\title{
Upregulating CXCR7 Accelerates Endothelial Progenitor Cells Mediated Endothelial Reparation via Activating Akt/Keap-1/Nrf2 Signaling in Diabetes Mellitus
}

\section{Chunyu Jiang}

Shanghai 9th Peoples Hospital Affiliated to Shanghai Jiaotong University School of Medicine

\section{Ruiting Li}

shanghai sixth peoples hospital

\section{Chaoyang Xiu}

: Shanghai Sixth Peoples Hospital

\section{Xu Ma}

Shanghai Sixth Peoples Hospital

Hui Hu

Shanghai Sixth Peoples Hospital

\section{Liming Wei}

Shanghai Sixth Peoples Hospital

\section{Yihan Tang}

Yangzhou University Medical Academy

\section{Mingyang Tao}

Yangzhou University Medical Academy

Jungong Zhao ( $\nabla$ zhaojungongradio@hotmail.com )

Shanghai Sixth Peoples Hospital

\section{Research}

Keywords: Diabetes mellitus, Endothelial progenitor cells, CXCR7, Nrf2, Reendothelialization, Neointimal hyperplasia

Posted Date: September 4th, 2020

DOI: https://doi.org/10.21203/rs.3.rs-70187/v1

License: (c) (i) This work is licensed under a Creative Commons Attribution 4.0 International License. Read Full License 


\section{Abstract}

Background: Dysfunction of endothelial progenitor cells (EPCs) contributes to vascular disease in diabetes mellitus. However, the molecular mechanism underlying dysfunction of endothelial progenitor cells in diabetes mellitus remains unclear. Our study was aimed to illustrate the potential molecular mechanism underlying diabetic EPCs dysfunction in vivo and vitro. Furthermore, to assess the effect of EPCs transplantation on endothelium regeneration in diabetic rats.

Methods: Functional assay in vitro and western blotting was conducted to reveal the association between C-X-C chemokine receptor type 7 (CXCR7) expression and diabetic EPCs dysfunction. To confirm the association between cellular CXCR7 level and EPCs function, the CXCR7 expression of EPCs was upregulated and downregulated via lentiviral transduction and RNA interference respectively. Western blotting was used to reveal the potential molecular mechanism underlying how Stromal-Derived Factor1(SDF-1)/CXCR7 pathway regulate EPCs function. To elucidate the role of SDF-1/CXCR7 axis in EPCs mediated endothelium regeneration, carotid artery injury model was built in diabetic rats. Then, saline, diabetic, normal or CXCR7 primed EPCs were injected into tail vein after carotid artery injury model was built.

Results: Diabetic EPCs dysfunction associated with decreasing CXCR7 expression. Furthermore, EPCs dysfunction can be mimicked by knockdown of CXCR7 in normal EPCs. However, upregulating CXCR7 expression can rescue diabetic EPCs dysfunction. SDF-1/CXCR7 axis positive regulate EPCs cellular function via activating AKT associated Kelch-like ECH-associated protein 1 (keap-1) / nuclear factor erythroid 2-related factor 2 (Nrf2) axis, which can be reversed by blockage of AKT and Nrf2. CXCR7-EPC transplantation, better than diabetic or normal EPCs, significantly accelerate endothelial repairing and attenuate neointimal hyperplasia in diabetes mellitus. But, the therapeutic effect of CXCR7-EPC transplantation on endothelium regeneration can be reversed by knockdown of Nrf2 molecules.

Conclusions: Diabetic EPCs dysfunction associated with decreasing CXCR7 expression. Furthermore, SDF-1/CXCR7 axis positive regulate EPCs cellular function via activating AKT/keap-1/Nrf2 axis. CXCR7 primed EPCs might be used for endothelial regeneration in diabetes associated vascular disease.

\section{Background}

Diabetes mellitus (DM) is a high-incidence, chronic, and metabolic disease causing many complications, such as peripheral and cardiovascular disease severely influencing patients' quality of life [1, 2]. Interventional therapy, especially stent implantation, has been widely used for DM-associated vascular events and has significantly improved the prognosis of patients. However, unfavorable events after stent implantation, such as in-stent restenosis and late stent thrombosis, remain a serious clinical challenge [3, 4]. A large body of evidence showed that endothelium injury caused by DM or interventional therapy contributed to these unfavorable vascular events [5-8]. Previous studies demonstrated that endothelial progenitor cells (EPCs) were vital in endothelial reparation [6-9]. However, DM impaired EPCs function 
and decreased the circulating EPCs level in either patients or mice with diabetes [10-12]. Thus, preventing adverse vascular events was crucial to understanding the potential mechanism underlying EPC dysfunction in DM.

Both CXCR4 and CXCR7 are receptors of SDF-1 (known as CXCL12) and expressed by EPCs [13-18]. The role of the SDF-1/CXCR4 axis in progenitor mobilization, recruitment, tube formation, and angiogenesis has been reported by previous studies $[14,15]$. CXCR7 is the second receptor that binds to SDF-1 with tenfold higher affinity compared with CXCR4 [16]. The SDF-1/CXCR7 axis is involved in regulating proliferation, adhesion, and angiogenesis of EPCs $[17,18]$. Moreover, dysfunctional EPCs derived from patients with hypertension have a lower CXCR7 level, which is associated with delayed reendothelialization [19]. All these findings indicate that DM may impair EPC functional activity via altering CXCR7 expression. However, this hypothesis remains unclear. The present study assessed the association between diabetic EPC dysfunction and cellular CXCR7 expression in DM.

The molecular mechanism underlying the regulation of cellular functional activity by the SDF-1/CXCR7 axis remains unclear. Nuclear factor erythroid 2-related factor 2 (Nrf2) is a critical redox sensor for oxidative stress $[20,21]$. Activated Nrf2, released from Kelch-like ECH-associated protein 1 (Keap-1, the repressor protein of Nrf2), translocates to the nucleus, binds to antioxidant response element (ARE), activates the transcription of target antioxidant genes, including Heme oxygenase - 1 (HO-1) and NADPH quinone oxidoreductase - 1 (NQO-1) [21, 22], and counteracts EPCs dysfunction caused by reactive oxygen species (ROS) $[23,24]$. Thus, it is hypothesized that the SDF-1/CXCR7 axis may regulate EPCs functional activity via activating the Akt-associated Keap-1/Nrf2 axis.

\section{Methods And Materials}

\section{EPC isolation and culture}

EPCs were isolated from the bone marrow of normal rats and rats with diabetes. The isolation, culture, and identification of EPCs were conducted as described in a previous study [25].

\section{Lentiviral transduction and RNA interference}

Lentiviral transduction and RNA interference were conducted to upregulate or downregulate the expression of CXCR7 so as to confirm the association between CXCR7 expression and EPC dysfunction.

Recombinant lentivirus encoding CXCR7 was constructed using a pLVX-EGFP-3FLAG-Puro vector (Shanghai Sunbio Medical Biotechnology, Shanghai, China). Briefly, EPCs were seeded into 24-well plates at a density of $1 \times 10^{5}$ cells/well and incubated overnight. EPCs were then transfected with purified lentiviral vectors that expressed recombinant CXCR7 overnight to achieve the multiplicity of infection of 25 with $3 \mu \mathrm{g} / \mathrm{mL}$ polybrene (Sigma, MO, USA). After $24 \mathrm{~h}$ of infection, the media was replaced with $2 \mathrm{~mL}$ of fresh media. Lentiviral transduction efficiency was determined by the lentivirus expressing green fluorescent protein (GFP). Apparent GFP expression was observed $48 \mathrm{~h}$ after transduction and reached 
the peak $72 \mathrm{~h}$ after transduction. The levels of CXCR7 expression were confirmed by Western blot analysis.

RNA interference was conducted to downregulate the expression of CXCR7 on EPCs. The sequences for RNA interference and negative control (NC) were as follows: CXCR7 siRNA sense: 5'-

GGAAGAUCAUCUUCUCCUATT-3', antisense: 5'-UAGGAGAAGAUGAUCUUCCGG-3'; NC: 5'UUCUCCGAACGUGUCACGUTT-3', antisense: 5'-ACGUGACACGUUCGGAGAATT-3'. Transfection was performed as described previously [26]. Briefly, siRNA transfection was performed using Lipofectamine 2000 reagent (Invitrogen, Carlsbad, CA, USA) following the manufacturer's protocols. EPCs were plated onto 6-well plates at a density of $5 \times 10^{5}$ cells/well overnight. The diluted siRNA and Lipofectamine 2000 reagent were mixed in a ratio of 1:1 (4 pmol siRNA to $4 \mu \mathrm{L}$ of Lipofectamine 2000) and incubated for 20 min at room temperature. Finally, $400 \mu \mathrm{L}$ was added to each well with a final volume of $2 \mathrm{~mL}$, and cells were continuously incubated for $48 \mathrm{~h}$ before starting the following experiments.

Nrf2 expression of CXCR7-primed EPCs was knocked down to reveal the detailed mechanism underlying the regulation of EPCs functional activity by the SDF-1/CXCR7 axis. Briefly, EPCs were infected with lentiviruses containing shRNA against Nrf2 or nonsense shRNA (Genomeditech, Shanghai, China). The sequences for $\mathrm{Nrf} 2$ and nonsense sequence were as follows:

Nrf2-shRNA 1: 5'- GGGTAAGTCGAGAAGTGTTTG -3'

Nrf2-shRNA 2: 5'- GGACCTAAAGCACAGCCAACA -3'

Nrf2-shRNA 3: 5'- GCAAGAAGCCAGATACAAAGA -3'

Nonsense shRNA: 5'- TTCTCCGAACGTGTCACGTAG -3'.

The transfection was performed following the previously described procedure. After transfection for $48 \mathrm{~h}$, the expression of Nrf2 was determined by Western blot analysis. Then, EPCs were infected with lentiviruses containing shRNA against Nrf2 with the best knockdown efficiency or nonsense shRNA following the previously described protocol.

\section{EPCs adhesion assay}

The adhesion of EPCs to human umbilical vein endothelial cells (HUVECs) was assessed by plating them in 24-well plates. First, HUVECs were plated on 24-well plates to form a monolayer. Then, nonattached cells were washed away with phosphate-buffered saline (PBS). DAPI $(10 \mu \mathrm{g} / \mathrm{mL})$ was used for staining HUVECs. EPCs were cultured in a medium containing Dil dye $(4 \mathrm{mg} / \mathrm{mL})$ for $30 \mathrm{~min}$ at $37^{\circ} \mathrm{C}$ following the manufacturer's protocol. Dil-labeled EPCs were digested and harvested after washing with PBS three times. Dil-labeled EPCs were then added to the plate, followed by incubation with the HUVEC monolayer for $2 \mathrm{~h}$ and washing of nonattached EPCs with PBS. Adherent EPCs were counted with five random fields under $400 \times$ magnification using an Olympus microscope. 


\section{Repairing capacity of EPCs in vitro}

Th repairing capacity of EPCs in vitro was assessed using the scratch assay. Briefly, EPCs were plated in 96-well plates to form a monolayer after 48-h culture. Then, the confluent monolayer was scratched using a p200 pipette tip $(1 \mathrm{~mL})$ containing serum-free media. The wound area was captured under a microscope (magnification, $\times 100$; Olympus Corporation) at baseline $(0 \mathrm{~h})$ and $24 \mathrm{~h}$ later and then analyzed using ImageJ software (National Institutes of Health).

\section{Western blot analysis}

EPCs were incubated for $24 \mathrm{~h}$ before protein extraction. EPCs proteins were extracted using a protein extraction kit (Solarbio, Beijing, China) and quantified using a bicinchoninic acid protein assay kit (Solarbio). Protein extracts were subjected to SDS-PAGE (KeyGEN, Nanjing, China) and then transferred onto polyvinylidene fluoride membranes (Roche, IN, USA). The following primary antibodies were used: anti-CXCR7 (1:1500), anti-GADPH (1:10000), anti-p-Akt (1:5000), Keap-1 (1:1000), anti-Nrf2 (1:1000), antiHO-1 (1:2000), and anti-NQO-1 (1:1000), which were obtained from Abcam (Life Technologies, CA, USA). Subsequently, the membranes were incubated with secondary antibodies (dilution, 1:1,000; Beijing Boaosen Biotechnology Co., Ltd.) for $2 \mathrm{~h}$ at room temperature. The protein bands were visualized using an Epson photo 1650 (Seiko Epson Corp. japan).

\section{Rat model and treatment regimens}

The vascular injury rat model was used to reveal the effect of putative EPCs on intimal reparation. Briefly, the type I diabetic rat model and vascular injury model were built as described previously [25]. Then, 1.5 $\mathrm{mL}$ of saline (group control) or cell suspension $\left(1 \times 10^{6}\right.$ cells $\left./ \mathrm{mL}\right)$ of normal EPCs (EPCs derived from normal rats, EPCs group), diabetic EPCs (EPCs derived from rats with diabetes, diabetic EPCs group), CXCR7-EPCs (EPCs derived from rats with diabetes, CXCR7-EPCs group), CXCR7-EPCs Nrf2-WT (EPCs derived from normal rats, CXCR7-EPCs ${ }^{\mathrm{Nrf2}-\mathrm{WT}}$ group), or CXCR7-EPCs Nrf2-KD (EPCs derived from normal

rats, CXCR7-EPCs ${ }^{\mathrm{Nrf2}-\mathrm{KD}}$ group) were injected into the circulation via the tail vein instantly and $12 \mathrm{~h}$ after establishing the vascular model.

\section{Assessment of reendothelialization and neointimal hyperplasia}

Reendothelialization was assessed on days 7 and 14 after the treatment using Evans blue staining. Briefly, $0.5 \mathrm{~mL}$ of $0.5 \%$ Evans blue dye was injected intravenously via the tail vein $30 \mathrm{~min}$ before sacrifice. Cardiac perfusion was then performed using formaldehyde fixation for $5 \mathrm{~min}$, followed by washing with PBS until the effluent was clearly run via the bilateral jugular vein. The common carotid artery was harvested $4 \mathrm{~mm}$ from the bifurcation and opened longitudinally. The blue-stained and unstained areas were measured in the entire injured region, and the reendothelialization rate (unstained area/total area) was then calculated. Digitalized images were analyzed using the NIH ImageJ 1.63 software. 
Neointimal hyperplasia was assessed using hematoxylin and eosin (HE) staining and Masson's staining on day 21 after the treatment. Denuded arteries were harvested from rats and embedded in formalin for $24 \mathrm{~h}$. Neointimal thickness was assessed using the intimal area-to-medial area ratio (I/M) in the axial sections stained with HE. A pathologist blinded to the treatment regimen assessed all the specimens using the $\mathrm{NIH}$ ImageJ 1.63 software.

\section{Statistical analysis}

Data were expressed as mean \pm standard deviation. One-way analysis of variance with post hoc LSD polynomial contrasts was used to determine significant differences between pairs of subgroups at one time point. $P$ values $<0.05$ were considered to be statistically significant.

\section{Results}

\section{Characteristics of EPCs}

EPCs clusters appeared 21 days later after the culture (Fig. 1a). Three markers (v-WF, CD34, and CD31) were used for detection by immunofluorescence to validate harvested EPCs (Fig. 1b). Flow cytometry showed that EPCs were positive for CD34, VEGFR2, and CD133 but negative for CD45 (Fig. 1C). In addition, acetylated low-density lipoprotein (ac-LDL) and endothelial-specific lectin UEA-1 showed positive staining in these cells (Fig. 1d), further confirming their identity as late EPCs.

\section{DM downregulated CXCR7 expression and attenuated the functional activity of EPCs}

Late EPCs were isolated from the bone marrow of normal rats and rats with diabetes to determine how DM affected the CXCR7 expression and functional activity of EPCs. Consequently, the expression of CXCR7 obviously decreased in EPCs derived from rats with diabetes compared with EPCs from normal rats (Fig. 2a and 2b). Meanwhile, the adhesion capacity of diabetic EPCs was attenuated compared with that of normal EPCs (normal group vs diabetic group: $23.8 \pm 4.9$ vs $9.4 \pm 1.2$ cells/field, $p=0.014$ ) (Fig. 2c and $2 \mathrm{~d}$ ). Furthermore, the scratch assay showed that the reparation capacity of EPCs also attenuated in vitro in DM (normal group vs diabetic group: $52.83 \pm 7.81 \%$ vs $24.93 \pm 3.69 \%, \mathrm{p}=0.038$; Fig. $2 \mathrm{e}$ and $2 \mathrm{f}$ ).

The gain- and loss-of-function studies were performed by siRNA knockdown in EPCs obtained from normal rats and overexpression in EPCs with lentivirus obtained from rats with diabetes to confirm the association between CXCR7 expression and EPC dysfunction. The study showed that EPC dysfunction was mimicked by the knockdown of CXCR7. Downregulating CXCR7 expression impaired the adhesion (normal group vs SiCXCR7 group: $23.8 \pm 4.9$ vs $10.7 \pm 1.1$ cells/field, $p=0.02$ ) and reparation capacity (normal group vs SiCXCR7 group: $52.83 \pm 7.81 \%$ vs $35.31 \pm 4.27 \%, \mathrm{p}=0.041$ ) of normal EPCs. In contrast, increasing CXCR7 expression significantly rescued the dysfunction of diabetic EPCs, including adhesion (diabetic group vs Lv-CXCR7 group: $9.4 \pm 1.2 v s 26.1 \pm 4.6$ cells/field, $p=0.011$ ) and reparation capacity in vitro (diabetic group vs Lv-CXCR7 group: $24.93 \pm 3.69 \%$ vs $67.5 \pm 8.49 \%, \mathrm{p}=0.026$; Fig. $2 \mathrm{c}-2 \mathrm{f}$ ). All these results indicated that diabetic EPC dysfunction was attributed to the decrease in CXCR7 expression. 


\section{Upregulating CXCR7 accelerated endothelial reparation of EPCs and attenuated neointimal hyperplasia in DM}

Saline-treated EPCs (control group), normal EPCs (normal group), diabetic EPCs (diabetic group), and CXCR7-primed EPCs (CXCR7 group) were transplanted into rats with diabetes after vascular injury to determine whether elevated CXCR7 levels accelerated EPC-mediated endothelial reparation.

Reendothelialization rate, I/M ratio, and neointimal area were used to assess endothelium regeneration and neointimal formation in different groups. CXCR7-EPC transplantation effectively accelerated reendothelialization on days 7 (control group vs CXCR7 group: $66.41 \pm 7.38 \%$ vs $94.66 \pm 9.6 \%, p=0.0298$ ) but not on days 14 (control group vs CXCR7 group: $86.46 \pm 10.35 \%$ vs $98.56 \pm 10.67 \%, p=0.398$ ) after vascular injury in DM (Fig. 3a-3c). Furthermore, the results showed that CXCR7-EPC transplantation significantly reduced neointimal formation at 21 days after treatment (control group vs CXCR7 group: I/M ratio: $75.63 \pm 10.45 \%$ vs $23.85 \pm 5.67 \%, \mathrm{p}<0.001$; neointimal area: $17.75 \pm 2.19$ vs $4.72 \pm 1.33, \mathrm{p}<0.001$ ). However, normal or diabetic EPC transplantation failed to accelerate endothelial repair and attenuate neointimal hyperplasia in DM (Fig. 3d and 3e). All these findings indicated that upregulating CXCR7 expression rescued diabetic EPCs dysfunction and accelerated EPCs-mediated endothelial reparation in DM.

\section{Elevation of CXCR7 stimulated HO-1 and NQO-1 expression via activating the Akt-associated Keap-1/Nrf2 axis}

The underlying mechanism by which the SDF-1/CXCR7 axis regulate EPCs function remains largely unknown. Previous studies showed that the Keap-1/Nrf2 axis was crucial in positively regulating EPC function via activating target antioxidant genes. Thus, it was hypothesized that the SDF-1/CXCR7 axis might positively regulate EPC function via activating the Akt-associated Keap-1/Nrf2 axis. To confirm this hypothesis, EPCs were obtained from the bone marrow of wild-type rats, and the expression levels of relevant signaling molecules, including p-Akt, Keap-1, Nrf2, HO-1, and NQO-1, were assessed by Western blot analysis.

The p-Akt level in CXCR7-EPCs was higher than that in normal EPCs after pretreatment with SDF-1. Moreover, pretreatment with SDF-1 significantly decreased the Keap-1 level but promoted the accumulation of nuclear Nrf-2 in CXCR7-EPCs compared with normal EPCs. The HO-1 and NOQ-1 levels were also higher in CXCR7-EPCs than in normal EPCs (Fig. 4a). Then, Akt molecules were blocked with LY29400 in normal EPCs and CXCR7-EPCs. The blockage of Akt molecules inactivated the Keap-1/Nrf2 axis and decreased the expression of its downstream target genes in both normal and CXCR7-EPCs (Fig. 4a). All these results indicated that the upregulation of CXCR7 controlled EPC functional activity via activating the Akt-associated Keap-1/Nrf2 axis.

\section{Knockdown of Nrf2 attenuated functional activity and endothelial reparation of CXCR7-EPCs}

Nrf2 molecules were knocked down with shRNA further to confirm the association between the Keap$1 /$ Nrf2 axis and the SDF-1/CXCR7 pathway. The knockdown of Nrf2 significantly decreased the Nrf2 
level, resulting in the reduction of HO-1 and NOQ-1 levels in both normal EPCs and CXCR7-EPCs (Fig. 4b). Meanwhile, the knockdown of Nrf2 blocked the protective effects of CXCR7 on EPCs functional activity in vitro (Fig. 5). Both adhesion capacity (CXCR7-EPCs ${ }^{\mathrm{Nrf2}-\mathrm{KD}}$ group vs CXCR7-EPCs ${ }^{\mathrm{Nrf2}-\mathrm{WT}}$ group: $15.94 \pm$ 2.78 vs $34.5 \pm 3.28$ cells/field, $\mathrm{p}=0.036$ ) and reparation capacity (CXCR7-EPCs ${ }^{\mathrm{Nrf2} 2-\mathrm{KD}}$ group vs CXCR7EPCs ${ }^{\text {Nrf2-WT }}$ group: $19.17 \pm 4.69 \%$ vs $37.54 \pm 6.42 \%, \mathrm{p}=0.029$ ) were significantly attenuated in CXCR7EPCs after the blockage of Nrf2 compared with those in CXCR7-EPCs (Fig. 5).

Saline-treated (control group), CXCR7-EPCs ${ }^{\mathrm{Nrf2}-\mathrm{WT}}$ (CXCR7-EPCs $^{\mathrm{Nrf2}-\mathrm{WT}}$ group), or CXCR7-EPCs ${ }^{\mathrm{Nrf2} 2 \mathrm{KD}}$ (CXCR7-EPCs ${ }^{\mathrm{Nrf2} 2 \mathrm{KD}}$ group) were transplanted into the carotid artery injury model of rats with diabetes to determine whether the knockdown of Nrf2 impaired CXCR7-EPC-mediated endothelial reparation in DM. Consequently, CXCR7-EPC ${ }^{\text {Nrf2-WT }}$ transplantation accelerated reendothelialization (CXCR7-EPC ${ }^{\text {Nrf2-WT }}{ }^{\text {vs }}$ control group: 7 days: $91.42 \pm 10.03 \%$ vs $52.19 \pm 4.55 \%, p<0.001 ; 14$ days: $97.05 \pm 11.86 \%$ vs $89.7 \pm$ $8.16 \%, \mathrm{p}=0.429$ ) and inhibited neointimal hyperplasia (CXCR7-EPC ${ }^{\mathrm{Nrf2}-W T} v s$ control group: I/M ratio: $23.85 \pm 5.67 \%$ vs $75.63 \pm 10.45 \%, \mathrm{p}<0.001$; neointimal area: $4.72 \pm 1.33$ vs $17.75 \pm 2.19 \times 10^{3} \mu \mathrm{m}^{2}, \mathrm{p}<$ $0.001)$ compared with the control group. However, the blockage of Nrf2 significantly impaired EPCsmediated endothelial reparation. The reendothelialization rate in the CXCR7-EPCs ${ }^{\mathrm{Nrf2}-\mathrm{KD}}$ group was lower than that in the CXCR7-EPCs ${ }^{\text {Nrf2-WT }}$ group at 7 days after treatment (CXCR7-EPC ${ }^{\text {Nrf2-WT }}$ vs CXCR7-

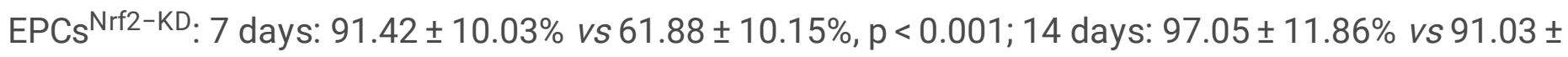
$10.52 \%, p=0.53)$. However, the reendothelialization rate showed no significant difference between the CXCR7-EPCs ${ }^{\mathrm{Nrf2} 2-K D}$ and control groups (CXCR7-EPC ${ }^{\mathrm{Nrf2}-K D}$ vs control group: 7 days: $61.88 \pm 10.15 \%$ vs $52.19 \pm 4.55 \%, p=0.426 ; 14$ days: $91.03 \pm 10.52 \%$ vs $89.7 \pm 8.16 \%, p=0.658$ ) (Fig. 6a-6c). Meanwhile, the I/M ratio in the CXCR7-EPCs ${ }^{\text {Nrf2-KD }}$ group was higher than that in CXCR7-EPCs ${ }^{\text {Nrf2-WT }}{ }^{\text {groups on day } 21}$

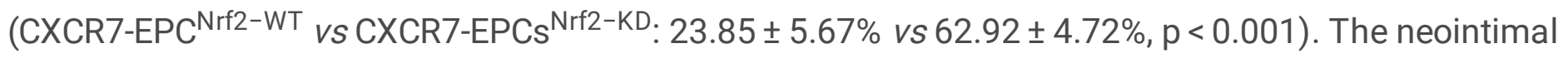
volume in the CXCR7-EPCs ${ }^{\text {Nrf2-KD }}$ group was also higher than that in CXCR7-EPCs ${ }^{\text {Nrf2-WT }}{ }^{\text {groups on day }}$ 21 (CXCR7-EPC ${ }^{\mathrm{Nrf2}-W T}$ vs CXCR7-EPCs ${ }^{\mathrm{Nrf2}-K D}: 4.72 \pm 1.33$ vs $15.86 \pm 2.12 \times 10^{3} \mu \mathrm{m}^{2}, \mathrm{p}<0.001$ ) (Fig. $6 \mathrm{~d}$ and $6 \mathrm{f}$ ). All these findings confirmed that the Keap-1/Nrf2 axis was downstream of the SDF-1/CXCR7 pathway, and was involved in regulating EPC function in vivo and in vitro.

\section{Discussion}

DM decreased the number of circulating EPCs and impaired EPCs functional activity [10-12], which were associated with the development and poor prognosis of vascular disease $[27,28]$. The present study revealed the molecular mechanism underlying DM-induced EPCs dysfunction. It had three novel findings. First, DM impaired EPCs functional activity associated with the downregulation of CXCR7 expression. Furthermore, the results showed that the SDF-1/CXCR7 pathway positively regulated EPCs function via activating the Akt/Keap-1/Nrf-2 axis. In addition, the transplantation of CXCR7-primed EPCs, rather than normal or diabetic EPCs, was an effective practice to promote endothelial reparation and inhibit neointimal hyperplasia in DM. 
Many studies showed that DM impaired EPCs cellular functions in humans and mice $[29,30]$. Some important molecules, such as CXCR4, NO, and p66Shc, have been identified as responsible for the dysfunction of cultured and circulating putative EPCs [11]. Several recent studies indicated that the SDF1/CXCR7 axis was involved in regulating EPCs cellular functions, including proliferation, adhesion, angiogenesis, and endothelial reparation in vitro or in vivo [17-19]. However, the number of relevant studies revealing the association between EPCs dysfunction and CXCR7 expression in DM was still inadequate. Similar to previous studies $[11,12,30]$, the present study also showed that the functional activity of EPCs derived from rats with diabetes was impaired significantly compared with that of EPCs from normal rats. Furthermore. the results confirmed the concept that decreasing CXCR7 expression contributed to EPCs dysfunction in DM. However, the detailed molecular mechanism by which diabetic EPCs downregulated CXCR7 expression is still unclear and needs further exploration.

Through dimerization with CXCR4, CXCR7 promotes cellular function via activating $\beta$-arrestin-dependent signaling [31, 32] and stimulating Akt phosphorylation [33]. However, the downstream mechanism by which the SDF-1/CXCR7 axis is involved in regulating EPC functional activity remains largely unknown. Wang et al. pointed out that Nrf2 protected EPCs against cellular dysfunction via decreasing cell senescence under hyperglycemic conditions [22]. It was also well documented that activated Nrf2 could bind to AREs and increase HO-1 and NQO-1 expression, thus exerting a cytoprotective effect via producing bioactive products or depleting intracellular sulfhydryl pools [20-21]. The results showed that the upregulation of CXCR7 stimulated Akt phosphorylation, Keap-1 inactivation, Nrf2 accumulation, and HO-1 and NQO-1 expression, accompanied by the augmentation of EPC functional activity, which was reversed by blocking Akt or Nrf2 molecules using LY2900 or ShRNA, respectively. All these results confirmed the concept that the SDF-1/CXCR7 axis positively modulated EPC functional activity via activating Keap-1/Nrf2-associated antioxidant genes, including HO-1 and NQO-1.

The levels of EPCs, which contributed to endothelial reparation via paracrine effect or transdifferentiating into mature endothelial cells [8], reduced significantly in patients with DM and other diabetes-related disorders. Transplantation of exogenous EPCs was considered as a potential method to promote endothelial reparation via elevating the circulating EPC level directly in a rabbit model [34]. However, the results of different studies were controversial [34-37]. Thus, the present study evaluated the role of EPCs in endothelial reparation. The results showed that CXCR7-primed EPCs transplantation was the best practice to promote reendothelialization in rats with diabetes. These results indicated that DM impaired the functional activity of normal EPCs but not of CXCR7-primed EPCs in vivo. The in vitro study showed that the main reason behind protecting CXCR7-EPCs against hyperglycemia-induced oxidative stress was that the upregulation of CXCR7 activated Nrf2-associated antioxidant genes, including HO-1 and NQO-1. The results were well supported by the findings of Dai et al., showing that upregulating CXCR7 expression completely inhibited the increased ROS level of CXCR7-EPCs exposed to ox-LDL or HG via stimulating HO1 and NQO-1 molecules [18]. In a word, the results showed that CXCR7-EPCs might serve a potential candidate for vascular regeneration in the future. 


\section{Conclusions}

As shown in Fig. 7, elevating CXCR7 expression preserved the functional activity of diabetic EPCs and protected against diabetes-associated oxidative stress damage via activating the Keap-1/Nrf2 axis in vivo and in vitro. Furthermore, the transplantation of CXCR7-EPCs was an effective practice to promote endothelial reparation, indicating that CXCR7-EPCs might be used for treating vascular disease in the future.

\section{Abbreviations}

EPCs: Endothelial progenitor cells

CXCR7: C-X-C chemokine receptor type 7

SDF-1: Stromal-Derived Factor-1

Keap-1: Kelch-like ECH-associated protein 1

Nrf2: Nuclear factor erythroid 2-related factor 2

DM: Diabetes mellitus

ARE: antioxidant response element

H0-1: Heme oxygenase -1

NQO-1: NADPH quinone oxidoreductase 1

ROS『reactive oxygen species

GFP囚green fluorescent protein

HUVEC囚human umbilical vein endothelial cell

\section{Declarations}

\section{Ethics approval}

The procedures involving animals were executed following the ethical standards of Guide for the Care and Use of Laboratory Animals and approved by shanghai sixth peoples hospital (No: 2016-0012).

\section{Consent for publication}

Not applicable 
The datasets used and analyzed in current study are available from the corresponding author based on reasonable request.

\section{Competing interest}

The authors declare that we have no competing interests.

\section{Funding}

This study was supported by the National Natural Science Foundation of China (Grant No. 81271683 and 81671791), and the Shanghai Key Discipline of Medical Imaging (Grant No. 2017ZZ02005).

\section{Authors' contributions}

CJ, XM, CX, RL, YT and MT performed the experiments. JZ, CX and CJ designed the experiments. RL, HH, LW, YT and MT conducted the statistical analysis of the data. CJ, XM, CX, RL, YT and MT drafted the manuscript. JZ, CJ, RL, CX conceived the study, participated in its design and coordination, and helped to draft the manuscript. All authors read and approved the final manuscript.

\section{Acknowledgements}

Not applicable.

\section{References}

1. Yang SL, Zhu LY, Han R, Sun LL, Li JX, Dou JT. Pathophysiology of peripheral arterial disease in diabetes mellitus. Journal of diabetes. 2017;9(2):133-40.

2. Glovaci D, Fan W, Wong ND. Epidemiology of Diabetes Mellitus and Cardiovascular Disease. Current cardiology reports. 2019;21(4):21.

3. Byrne RA, Joner M, Kastrati A. Stent thrombosis and restenosis: what have we learned and where are we going? The Andreas Gruntzig Lecture ESC 2014. European heart journal. 2015;36(47):3320-31.

4. Jukema JW, Ahmed TA, Verschuren JJ, Quax PH. Restenosis after PCI. Part 2: prevention and therapy. Nature reviews Cardiology. 2011;9(2):79-90.

5. Kipshidze N, Dangas G, Tsapenko M, Moses J, Leon MB, Kutryk M, et al. Role of the endothelium in modulating neointimal formation: vasculoprotective approaches to attenuate restenosis after percutaneous coronary interventions. Journal of the American College of Cardiology. 2004;44(4):7339.

6. Lim WH, Seo WW, Choe W, Kang CK, Park J, Cho HJ, et al. Stent coated with antibody against vascular endothelial-cadherin captures endothelial progenitor cells, accelerates re-endothelialization, and reduces neointimal formation. Arteriosclerosis, thrombosis, and vascular biology. 2011;31(12):2798-805. 
7. Padfield GJ, Newby DE, Mills NL. Understanding the role of endothelial progenitor cells in percutaneous coronary intervention. Journal of the American College of Cardiology. 2010;55(15):1553-65.

8. Simard T, Jung RG, Motazedian P, Di Santo P, Ramirez FD, Russo JJ, et al. Progenitor Cells for Arterial Repair: Incremental Advancements towards Therapeutic Reality. Stem cells international. 2017;2017:8270498.

9. Lan H, Wang Y, Yin T, Wang Y, Liu W, Zhang X, et al. Progress and prospects of endothelial progenitor cell therapy in coronary stent implantation. Journal of biomedical materials research Part B, Applied biomaterials. 2016;104(6):1237-47.

10. Arcangeli A, Lastraioli E, Piccini B, D'Amico M, Lenzi L, Pillozzi S, et al. Circulating Endothelial Progenitor Cells in Type 1 Diabetic Patients: Relation with Patients' Age and Disease Duration. Frontiers in endocrinology. 2017;8:278.

11. Wils J, Favre J, Bellien J. Modulating putative endothelial progenitor cells for the treatment of endothelial dysfunction and cardiovascular complications in diabetes. Pharmacology \& therapeutics. 2017;170:98-115.

12. Gallagher KA, Liu ZJ, Xiao M, Chen H, Goldstein LJ, Buerk DG, et al. Diabetic impairments in NOmediated endothelial progenitor cell mobilization and homing are reversed by hyperoxia and SDF-1 alpha. The Journal of clinical investigation. 2007;117(5):1249-59.

13. Doring Y, Pawig L, Weber C, Noels H. The CXCL12/CXCR4 chemokine ligand/receptor axis in cardiovascular disease. Frontiers in physiology. 2014;5:212.

14. Petit I, Jin D, Rafii S. The SDF-1-CXCR4 signaling pathway: a molecular hub modulating neoangiogenesis. Trends in immunology. 2007;28(7):299-307.

15. Zhao Z, Ma X, Ma J, Sun X, Li F, Lv J. Naringin enhances endothelial progenitor cell (EPC) proliferation and tube formation capacity through the CXCL12/CXCR4/PI3K/Akt signaling pathway. Chemico-biological interactions. 2018;286:45-51.

16. Balabanian K, Lagane B, Infantino S, Chow KY, Harriague J, Moepps B, et al. The chemokine SDF$1 / C X C L 12$ binds to and signals through the orphan receptor RDC1 in T lymphocytes. The Journal of biological chemistry. 2005;280(42):35760-6.

17. Dai $X$, Tan $Y$, Cai S, Xiong $X$, Wang L, Ye Q, et al. The role of CXCR7 on the adhesion, proliferation and angiogenesis of endothelial progenitor cells. Journal of cellular and molecular medicine. 2011;15(6):1299-309.

18. Dai X, Yan X, Zeng J, Chen J, Wang Y, Chen J, et al. Elevating CXCR7 Improves Angiogenic Function of EPCs via Akt/GSK-3beta/Fyn-Mediated Nrf2 Activation in Diabetic Limb Ischemia. Circulation research. 2017;120(5):e7-e23.

19. Zhang XY, Su C, Cao Z, Xu SY, Xia WH, Xie WL, et al. CXCR7 upregulation is required for early endothelial progenitor cell-mediated endothelial repair in patients with hypertension. Hypertension. 2014;63(2):383-9. 
20. Loboda A, Damulewicz M, Pyza E, Jozkowicz A, Dulak J. Role of Nrf2/HO-1 system in development, oxidative stress response and diseases: an evolutionarily conserved mechanism. Cellular and molecular life sciences : CMLS. 2016;73(17):3221-47.

21. Leinonen HM, Kansanen E, Polonen P, Heinaniemi M, Levonen AL. Role of the Keap1-Nrf2 pathway in cancer. Advances in cancer research. 2014;122:281-320.

22. Wang RY, Liu LH, Liu H, Wu KF, An J, Wang Q, et al. Nrf2 protects against diabetic dysfunction of endothelial progenitor cells via regulating cell senescence. International journal of molecular medicine. 2018;42(3):1327-40.

23. Sorrentino SA, Bahlmann FH, Besler C, Muller M, Schulz S, Kirchhoff N, et al. Oxidant stress impairs in vivo reendothelialization capacity of endothelial progenitor cells from patients with type 2 diabetes mellitus: restoration by the peroxisome proliferator-activated receptor-gamma agonist rosiglitazone. Circulation. 2007;116(2):163-73.

24. Callaghan MJ, Ceradini DJ, Gurtner GC. Hyperglycemia-induced reactive oxygen species and impaired endothelial progenitor cell function. Antioxidants \& redox signaling. 2005;7(11-12):1476-82.

25. Li X, Chen C, Wei L, Li Q, Niu X, Xu Y, et al. Exosomes derived from endothelial progenitor cells attenuate vascular repair and accelerate reendothelialization by enhancing endothelial function. Cytotherapy. 2016;18(2):253-62.

26. Long P, Sun F, Ma Y, Huang Y. Inhibition of CXCR4 and CXCR7 for reduction of cell proliferation and invasion in human endometrial cancer. Tumour biology : the journal of the International Society for Oncodevelopmental Biology and Medicine. 2016;37(6):7473-80.

27. Hill JM, Zalos G, Halcox JP, Schenke WH, Waclawiw MA, Quyyumi AA, et al. Circulating endothelial progenitor cells, vascular function, and cardiovascular risk. The New England journal of medicine. 2003;348(7):593-600.

28. Werner N, Kosiol S, Schiegl T, Ahlers P, Walenta K, Link A, et al. Circulating endothelial progenitor cells and cardiovascular outcomes. The New England journal of medicine. 2005;353(10):999-1007.

29. Han $X$, Tao Y, Deng Y, Yu J, Sun Y, Jiang G. Metformin accelerates wound healing in type 2 diabetic db/db mice. Molecular medicine reports. 2017;16(6):8691-8.

30. Hu L, Dai SC, Luan X, Chen J, Cannavicci A. Dysfunction and Therapeutic Potential of Endothelial Progenitor Cells in Diabetes Mellitus. Journal of clinical medicine research. 2018;10(10):752-7.

31. Decaillot FM, Kazmi MA, Lin Y, Ray-Saha S, Sakmar TP, Sachdev P. CXCR7/CXCR4 heterodimer constitutively recruits beta-arrestin to enhance cell migration. The Journal of biological chemistry. 2011;286(37):32188-97.

32. Levoye A, Balabanian K, Baleux F, Bachelerie F, Lagane B. CXCR7 heterodimerizes with CXCR4 and regulates CXCL12-mediated G protein signaling. Blood. 2009;113(24):6085-93.

33. Tarnowski M, Liu R, Wysoczynski M, Ratajczak J, Kucia M, Ratajczak MZ. CXCR7: a new SDF-1binding receptor in contrast to normal CD34(+) progenitors is functional and is expressed at higher level in human malignant hematopoietic cells. European journal of haematology. 2010;85(6):472-83. 
34. Hu CH, Ke X, Chen K, Yang DY, Du ZM, Wu GF. Transplantation of human umbilical cord-derived endothelial progenitor cells promotes re-endothelialization of the injured carotid artery after balloon injury in New Zealand white rabbits. Chinese medical journal. 2013;126(8):1480-5.

35. Schachinger V, Erbs S, Elsasser A, Haberbosch W, Hambrecht R, Holschermann H, et al. Intracoronary bone marrow-derived progenitor cells in acute myocardial infarction. The New England journal of medicine. 2006;355(12):1210-21.

36. Traverse JH, Henry TD, Pepine CJ, Willerson JT, Zhao DX, Ellis SG, et al. Effect of the use and timing of bone marrow mononuclear cell delivery on left ventricular function after acute myocardial infarction: the TIME randomized trial. Jama. 2012;308(22):2380-9.

37. Surder D, Manka R, Lo Cicero V, Moccetti T, Rufibach K, Soncin S, et al. Intracoronary injection of bone marrow-derived mononuclear cells early or late after acute myocardial infarction: effects on global left ventricular function. Circulation. 2013;127(19):1968-79.

\section{Figures}

a

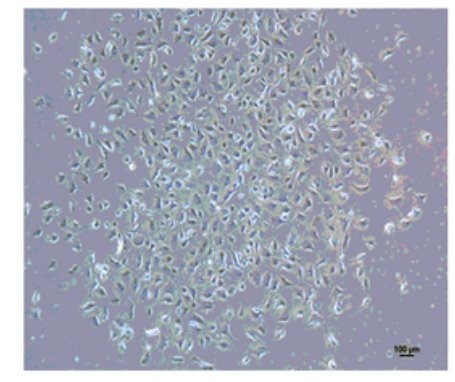

c

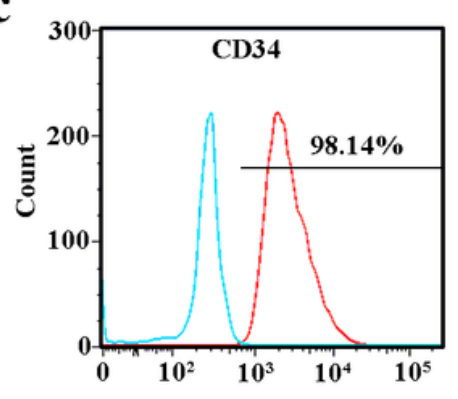

d

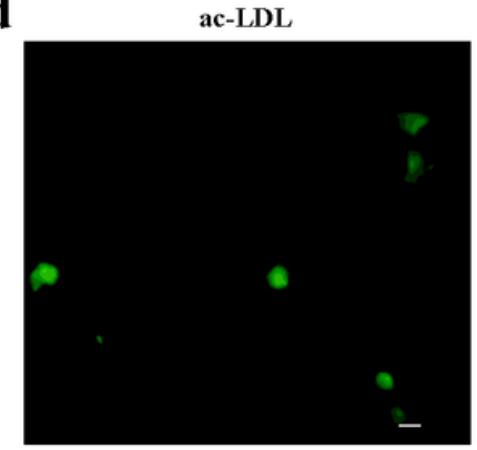

b
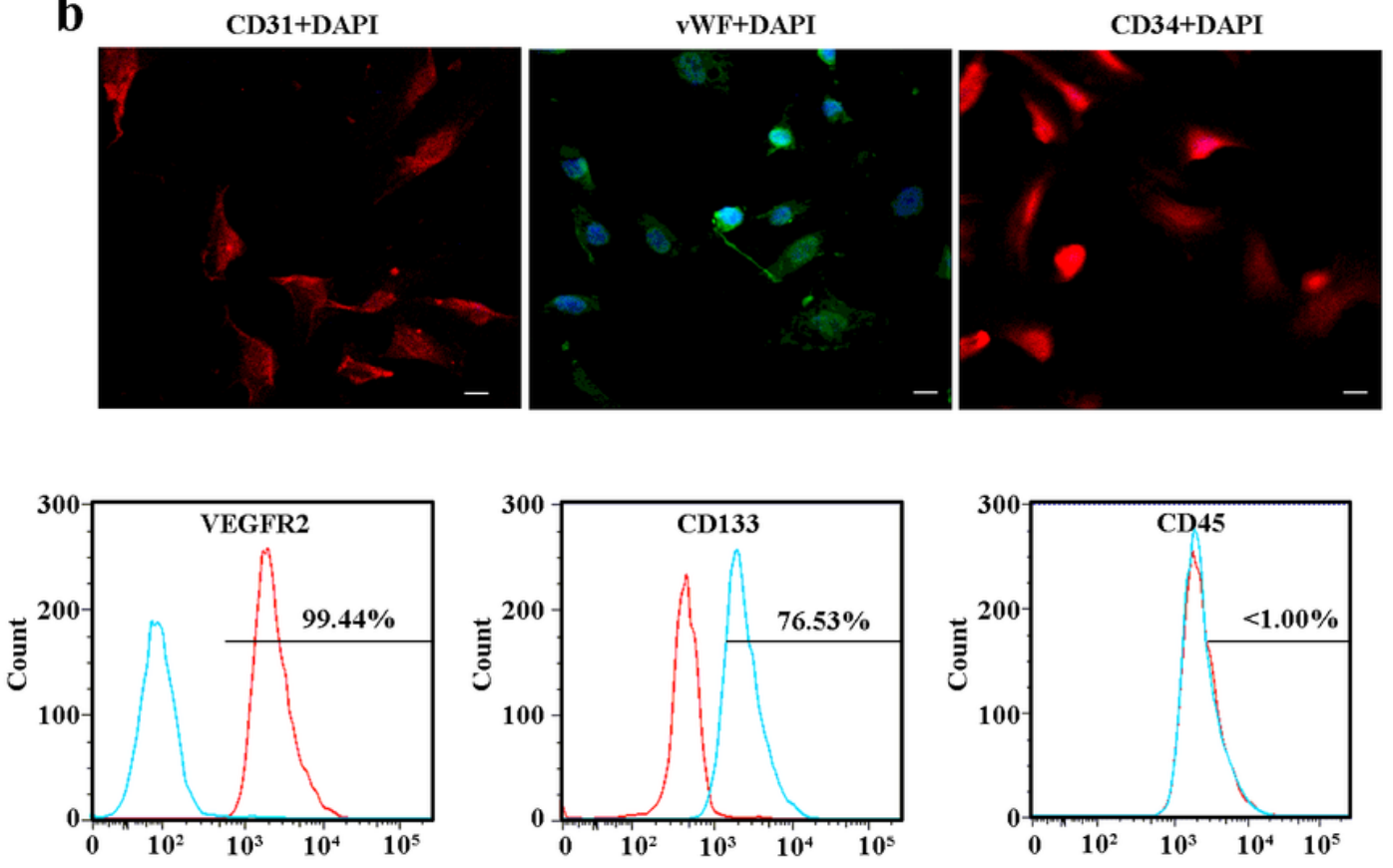

UEA-I

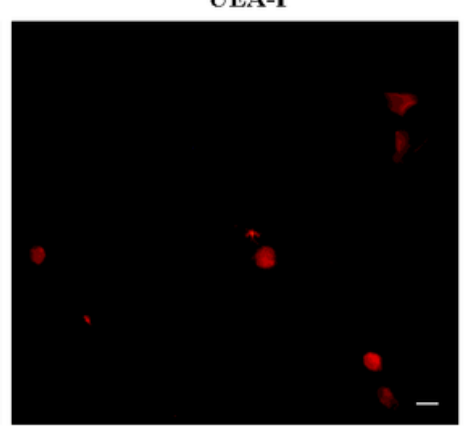

DAPI

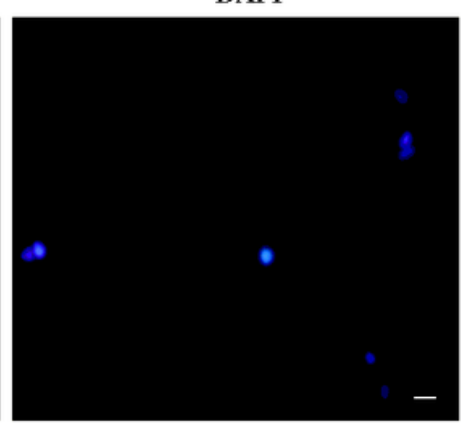

Merge

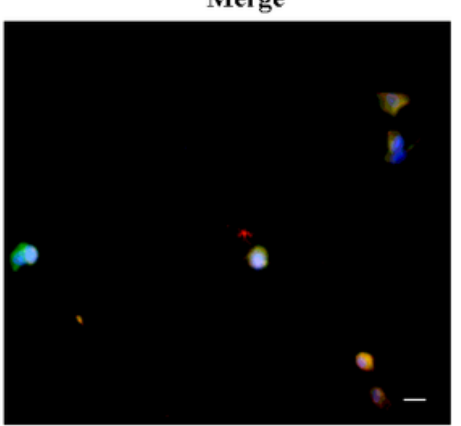


Figure 1

EPC characteristics. (a) EPCs showing typical endothelial-like cobblestone morphology after culturing for 21 days. (b) Immunofluorescence showed that EPCs were positive for v-WF, CD34, and CD31 staining (bar $=150 \mu \mathrm{m})$. (c) Flow cytometry showed that EPCs were positive for CD34, VEGFR2, and CD133 but negative for CD45. (d) EPCs could uptake ac-LDL and bind to UEA-1 (bar $=100 \mu \mathrm{m})$.

$\mathbf{a}$

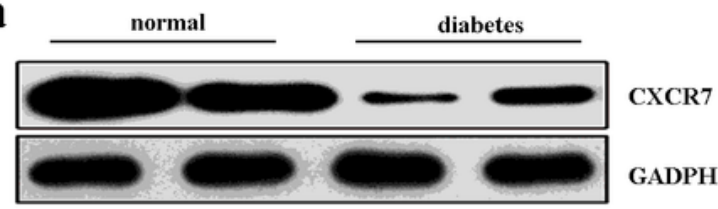

b

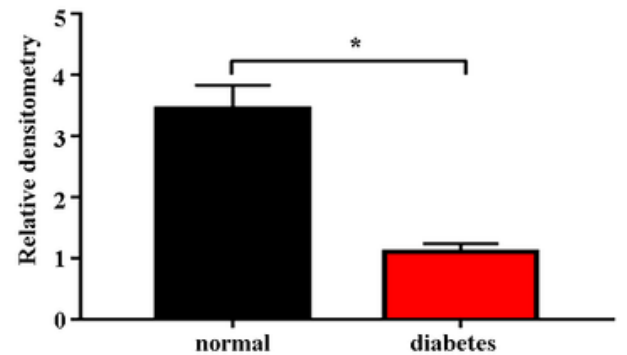

c

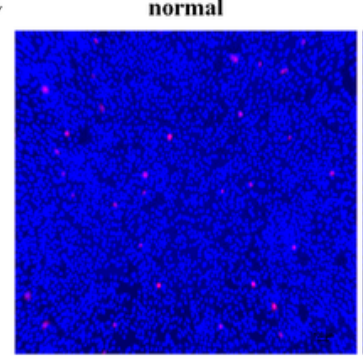

SiCXCR7

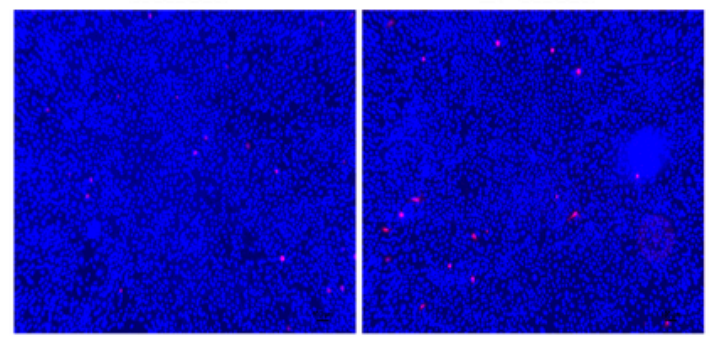

d

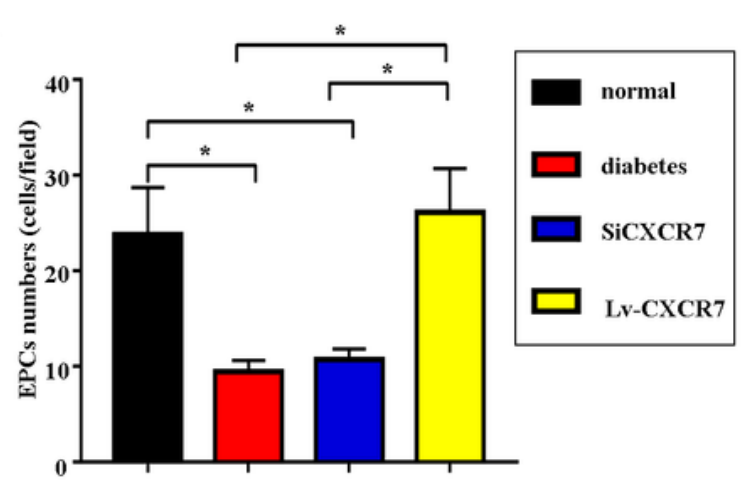

$\mathbf{e}$

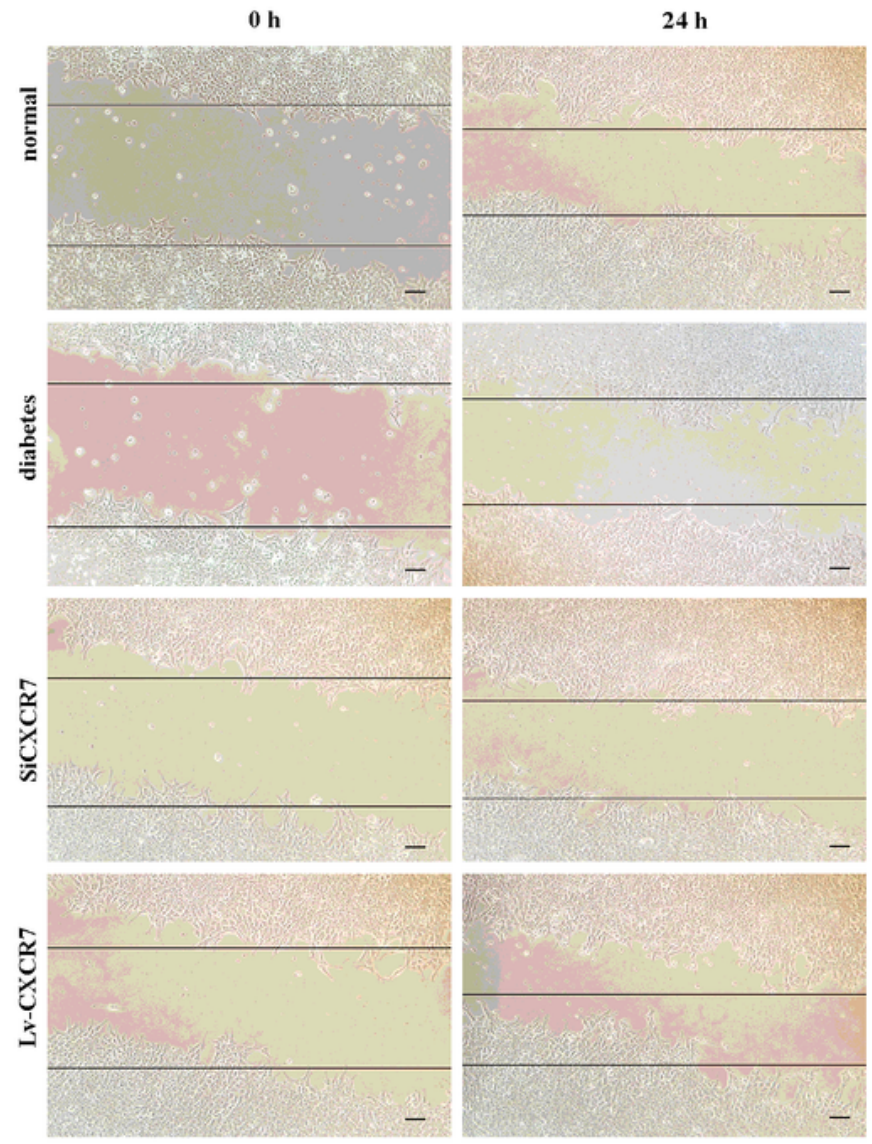

f

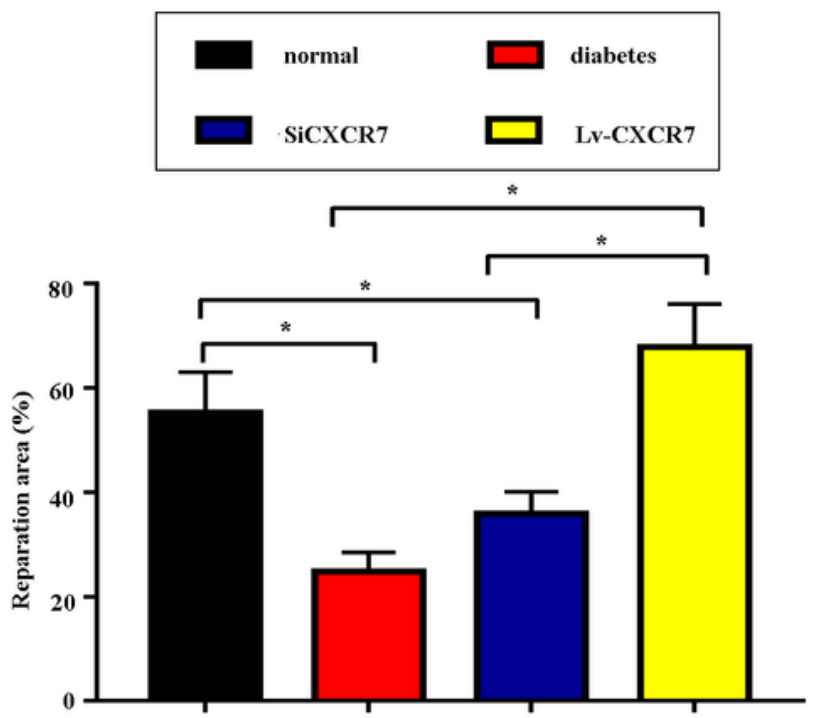

Figure 2 
Elevating CXCR7 expression enhanced the functional activity of EPCs. ( $a$ and b) XCR7 expression in diabetic EPCs (diabetic group) and normal EPCs (normal group) $(n=5)$. ( $c$ and d) Capacity to adhere to HUVECs in different groups (bar $=100 \mu \mathrm{m} ; \mathrm{n}=5$ ). (e and $\mathrm{f}$ ) Reparation capacity of EPCs in different groups (bar $=150 \mu m ; n=5)(* P<0.05)$.
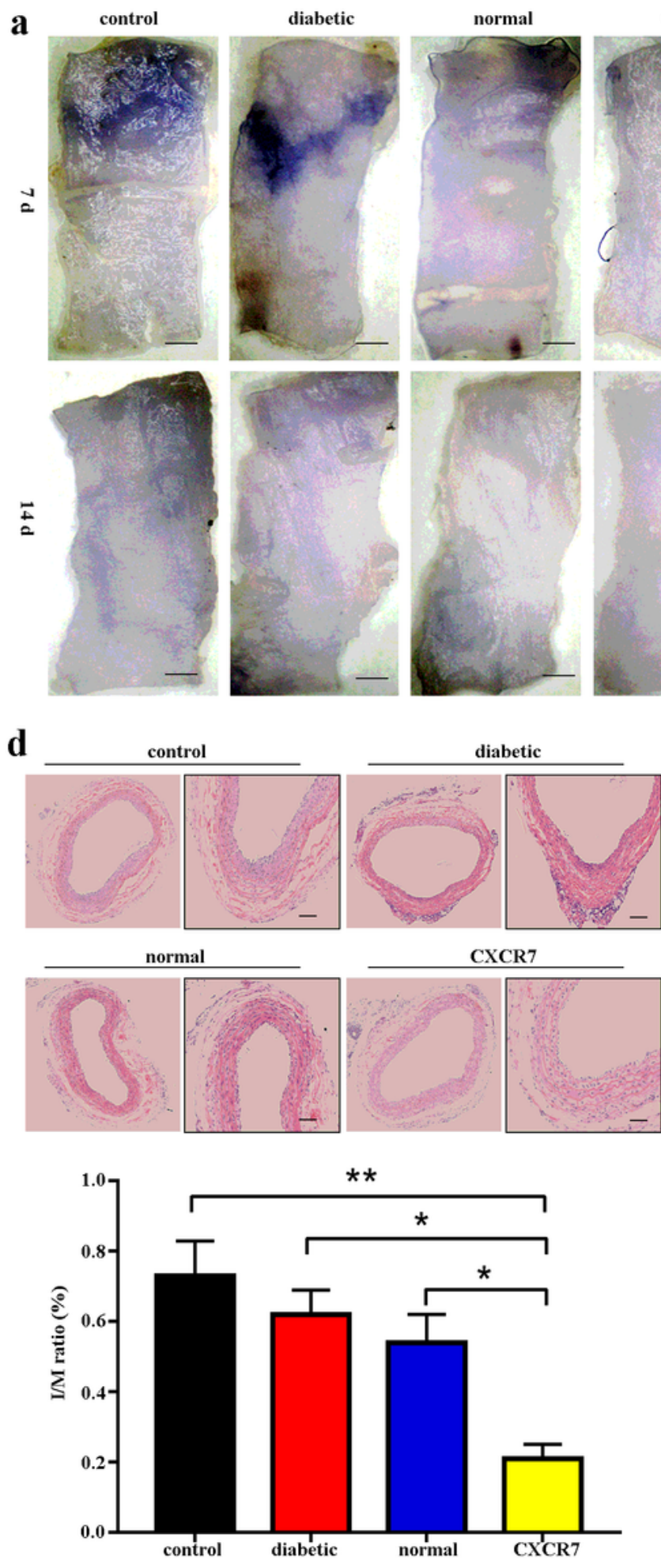

diabetic
CXCR7
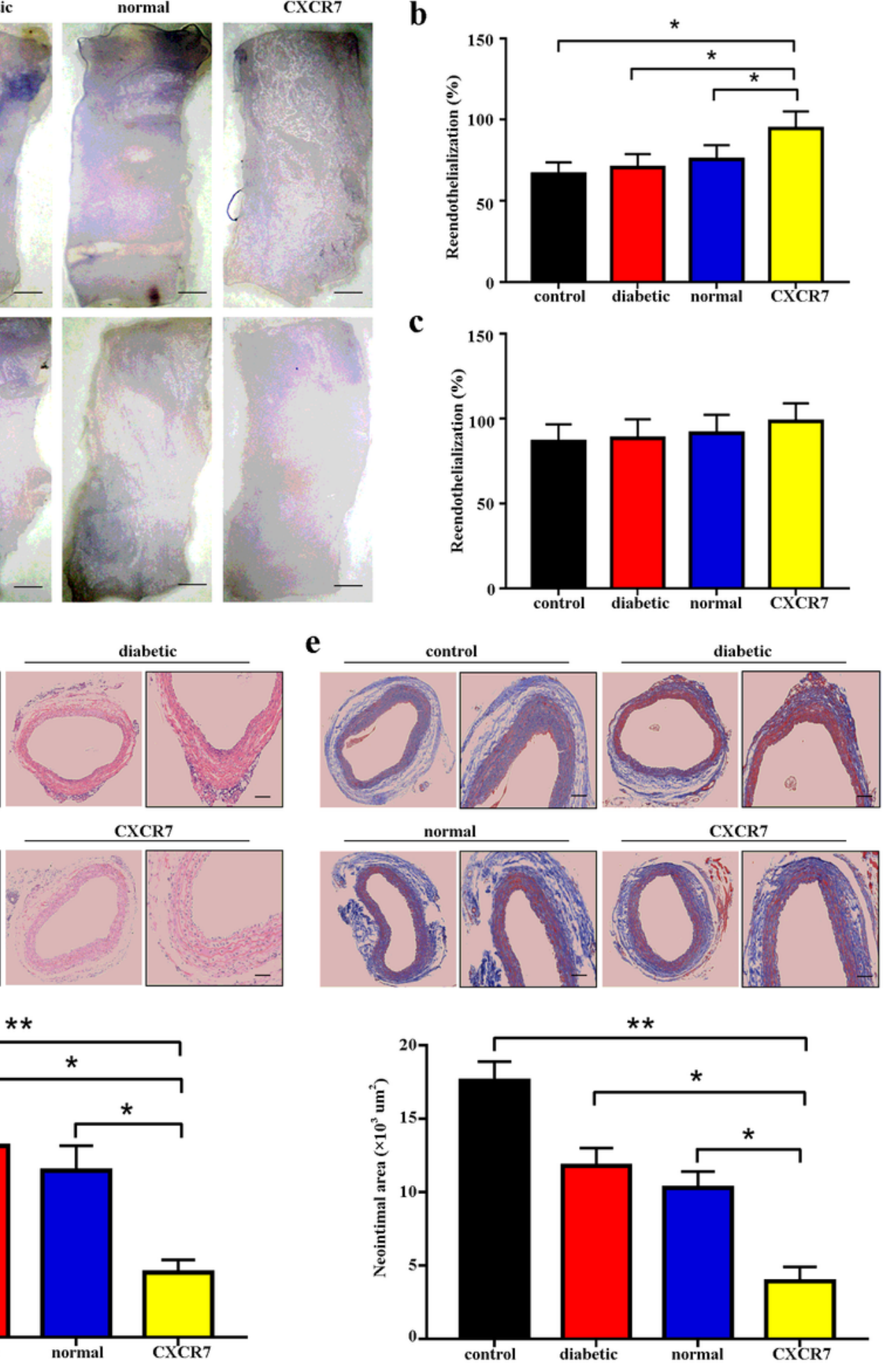

Figure 3 
Upregulating CXCR7 expression accelerated reendothelialization and inhibited neointimal hyperplasia. (a) Representative images of Evans blue staining (bar $=500 \mu \mathrm{m} ; n=5$ ). (b) Reendothelialization rate in different groups after 7 days. (c) Reendothelialization rate after 14 days. (d) I/M ratio of HE staining 21 days after treatment $(\mathrm{bar}=150 \mu \mathrm{m} ; \mathrm{n}=5)$. (e) Neointimal area with Masson staining 21 days after treatment (bar $=150 \mu m ; n=5) .\left({ }^{*} P<0.05 ; * * P<0.001\right)$.

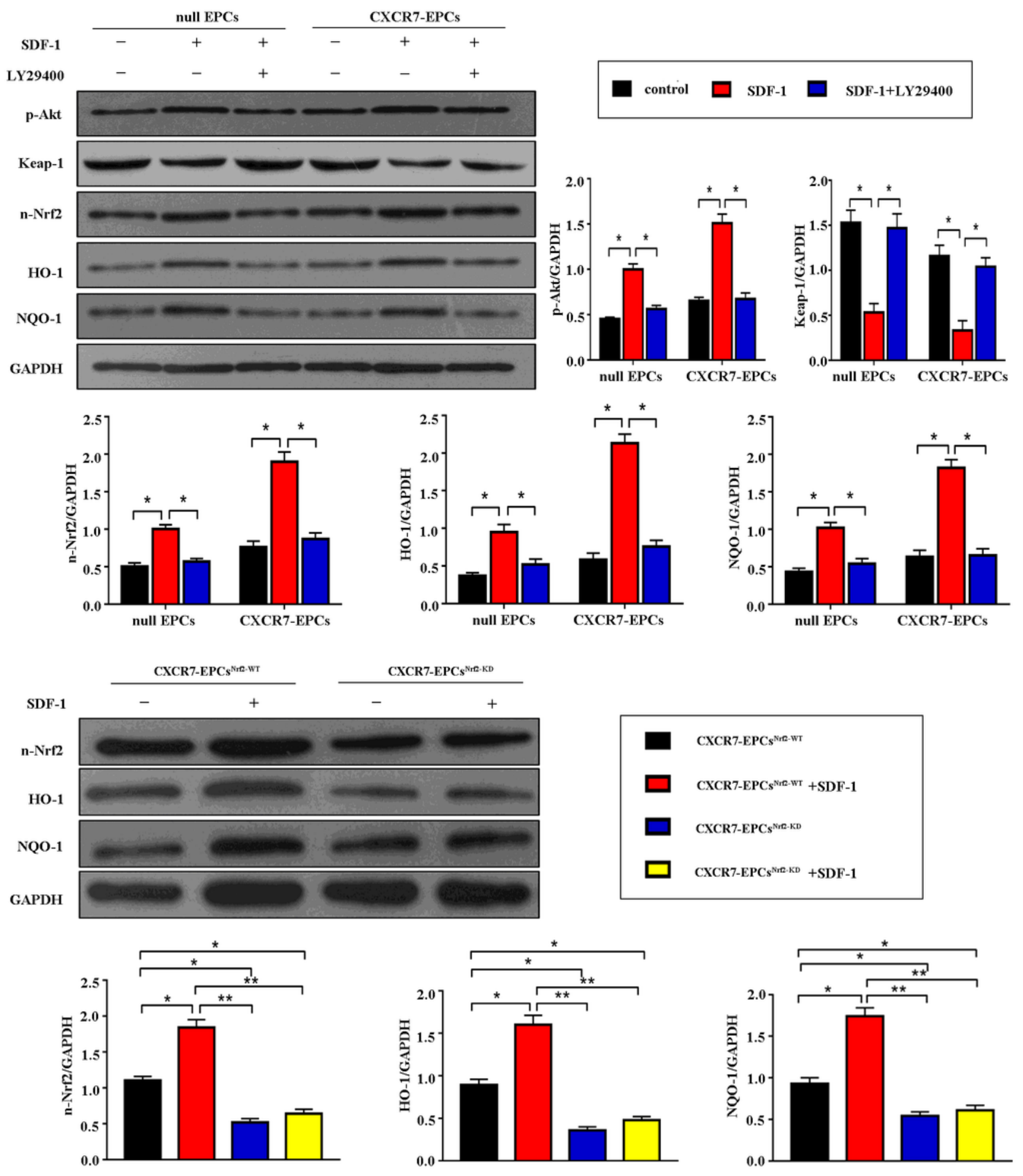

Figure 4 
Upregulating CXCR7 expression activated the Akt-associated Keap-1/Nrf2 axis. (a) Expression of p-Akt, Keap-1, n-Nrf2, HO-1, and NQO-1. (b) Expression of HO-1 and NQO-1 after the blockage of Nrf2 ( ${ }^{\mathrm{P}}<0.05$; $* * \mathrm{P}<0.001)$.

a

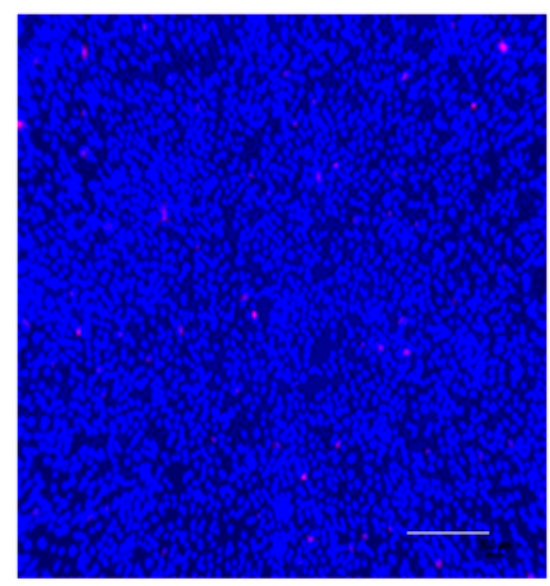

b

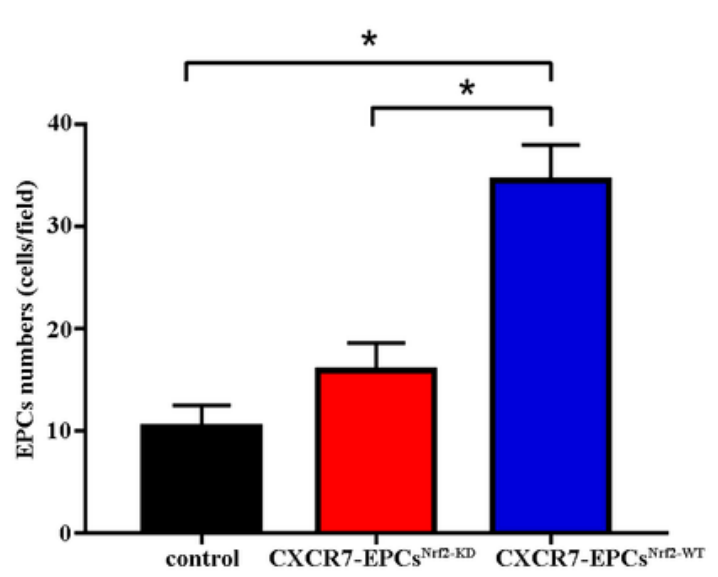

CXCR7-EPCs ${ }^{\mathrm{Nr} / \mathrm{KD}}$

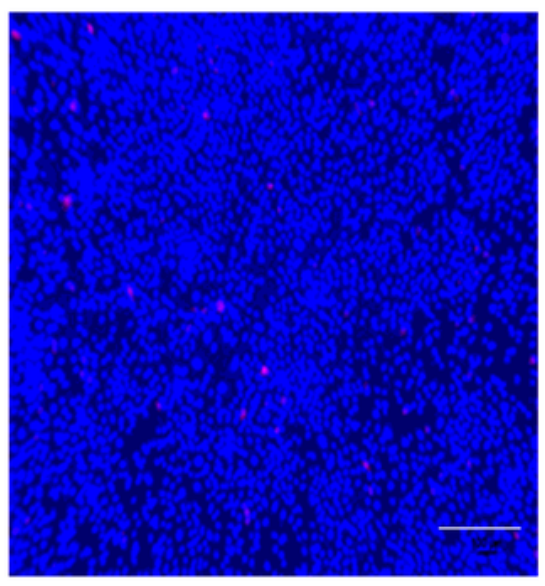

d

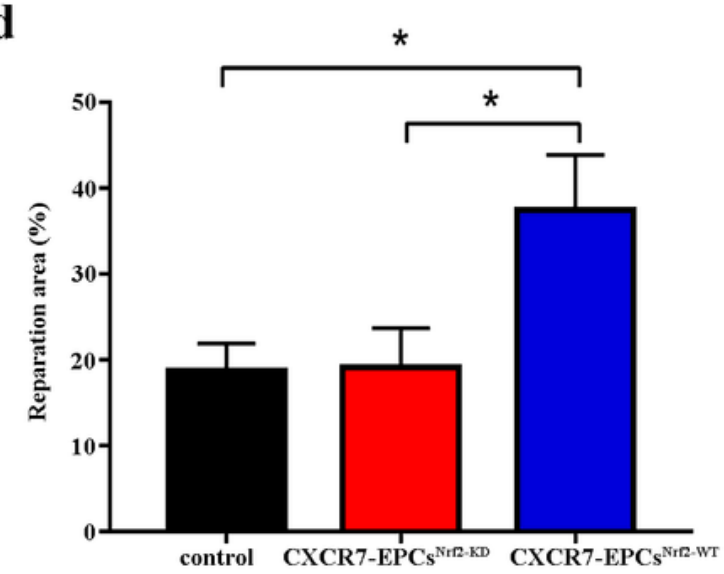

CXCR7-EPCs ${ }^{\mathrm{Nrr}-\mathrm{wT}}$

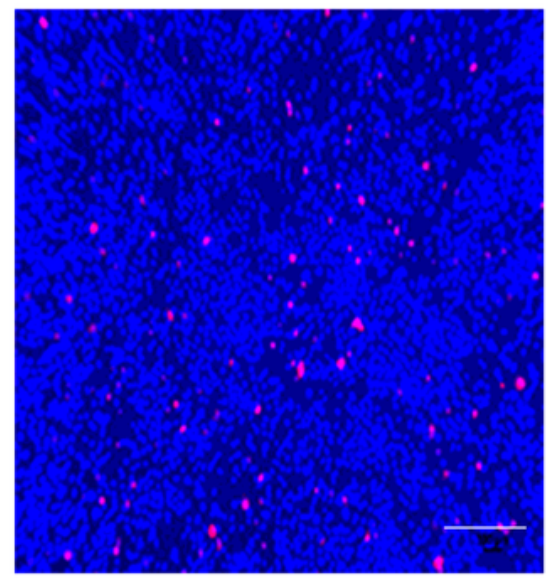

CXCR7-EPCs ${ }^{\mathrm{NrR}-\mathrm{KD}} \mathrm{CXCR}$ 7-EPCs ${ }^{\mathrm{Nr} 2-\mathrm{WT}}$ c

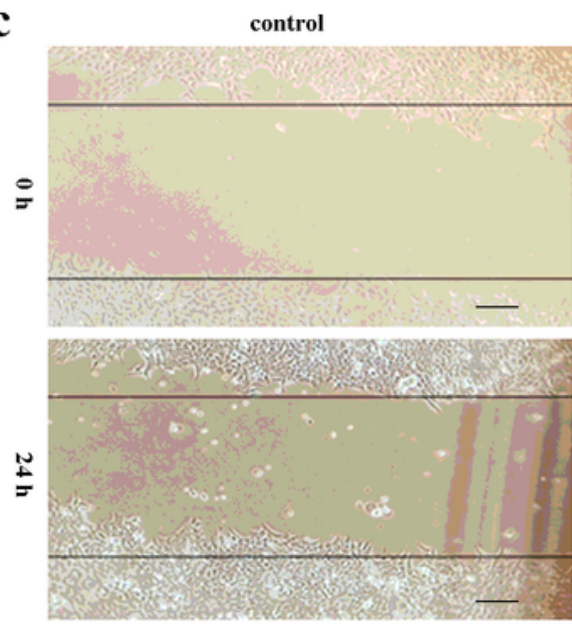

CXCR7-EPCs ${ }^{\mathrm{Nr} 2-\mathrm{KD}}$

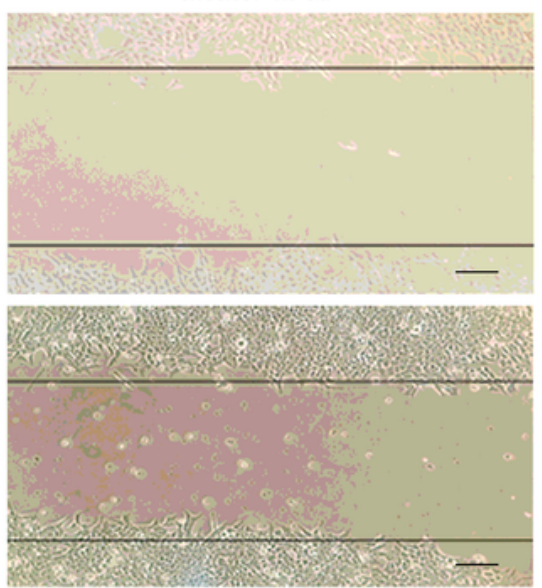

\section{Figure 5}

Knockdown of Nrf2 attenuated the functional activity of CXCR7-EPCs. (a and b) Difference in adhesion capacity among all groups (bar $=200 \mu m ; n=5)$. (c and d) Difference in reparation capacity among all 
groups (bar $=100 \mu m ; n=5)(* P<0.05)$.

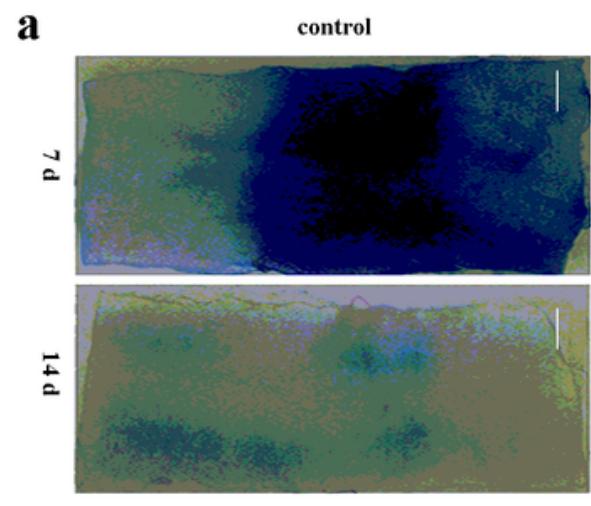

b

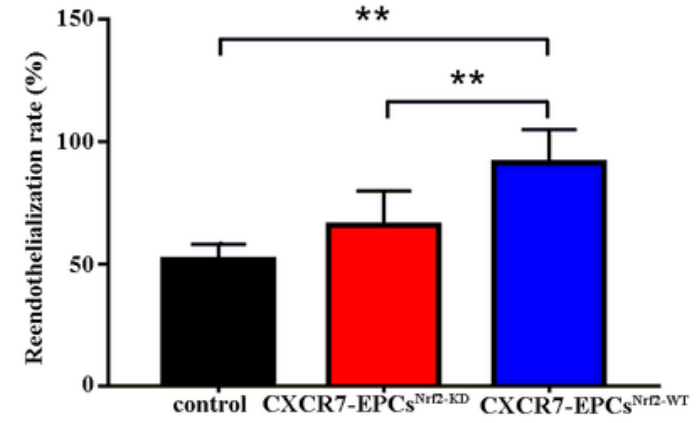

CXCR7-EPCs ${ }^{\mathrm{Nr} 2-\mathrm{KD}}$
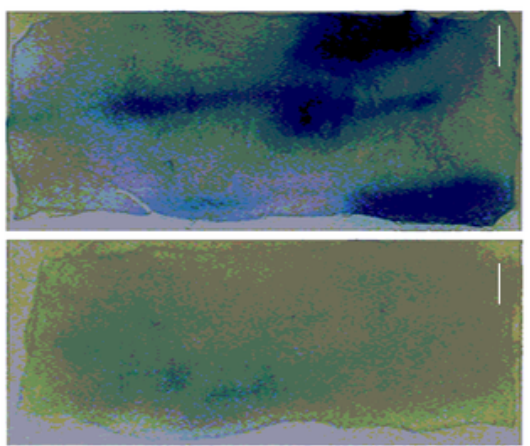

c

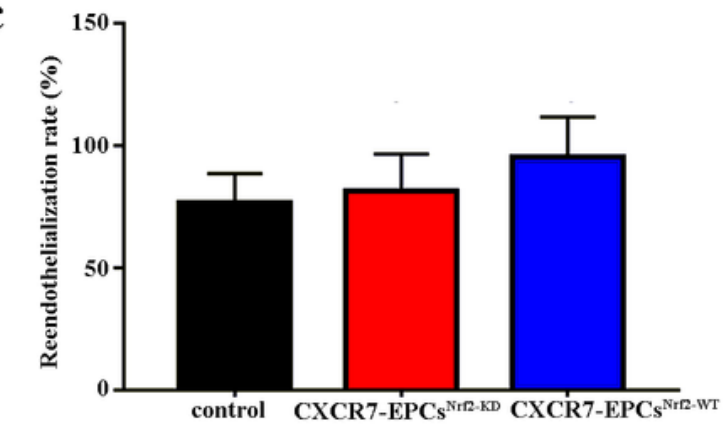

d
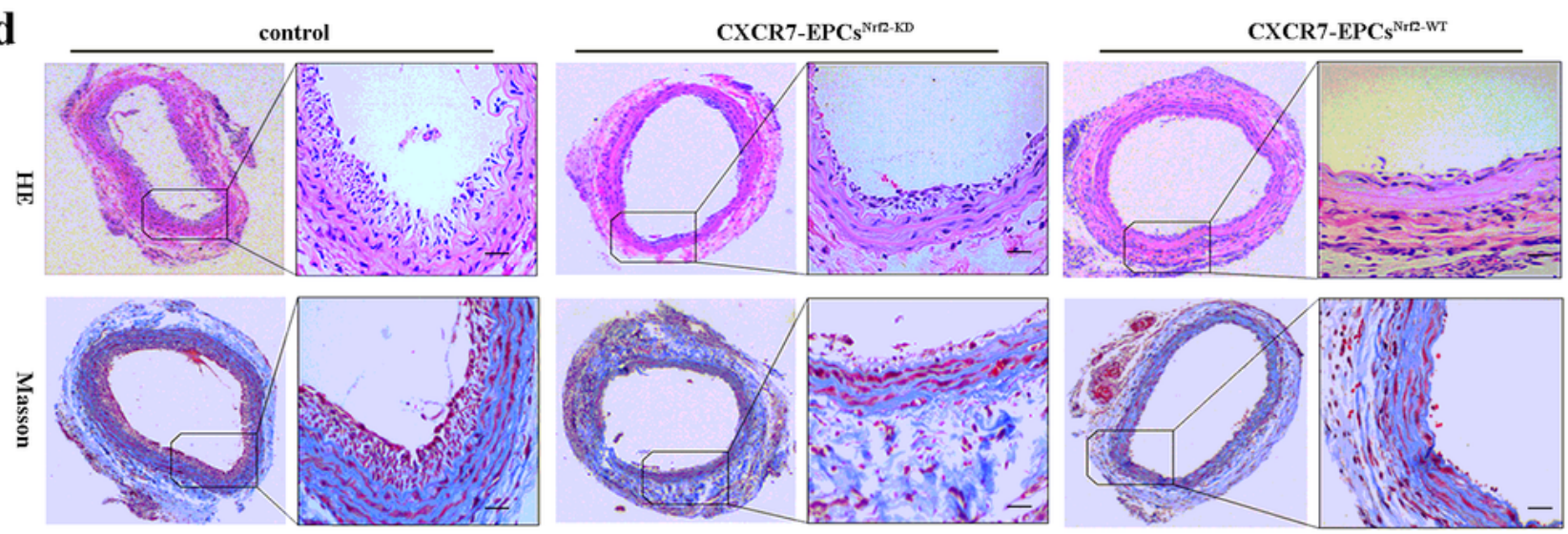

$\mathbf{e}$
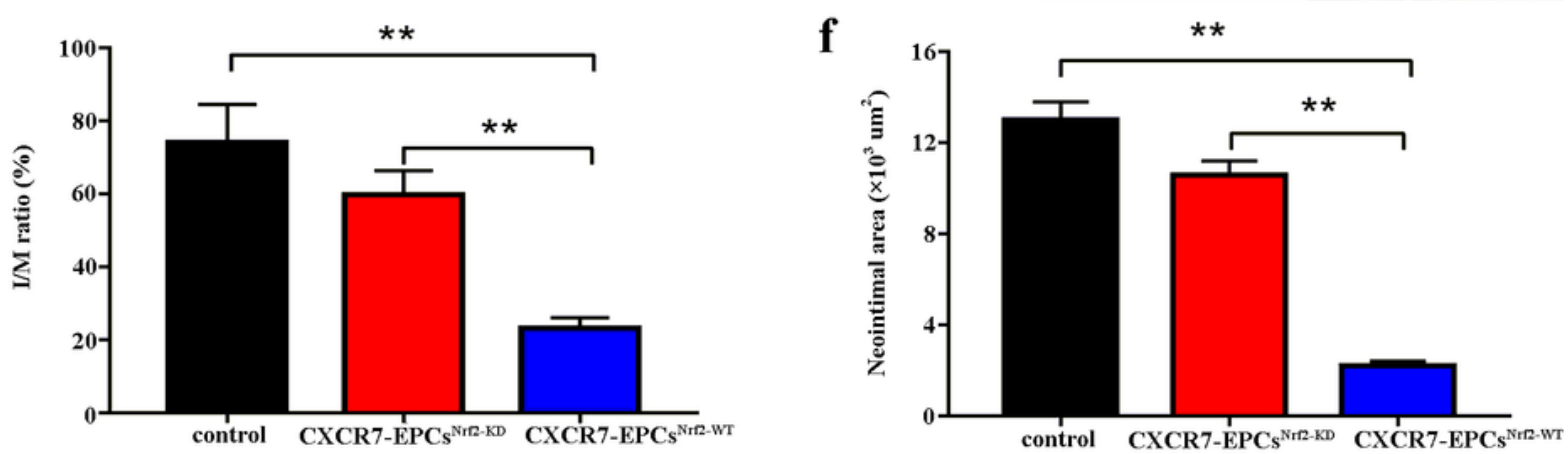

Figure 6

Knockdown of Nrf2 impaired endothelial reparation of CXCR7-EPCs in vivo. (a) Representative images of Evans blue staining after EPC transplantation (bar $=500 \mu \mathrm{m} ; \mathrm{n}=5$ ). (b) Reendothelialization rate in different groups after 7 days. (c) Reendothelialization rate in different groups after 14 days. (d) Representative images of HE and Masson staining (bar $=150 \mu \mathrm{m} ; \mathrm{n}=5)$. (e) I/M ratio in different groups 
after EPC transplantation. (f) Neointimal area in different groups after EPC transplantation $\left({ }^{\star} P<0.05 ; \star \star P\right.$ $<0.001)$.

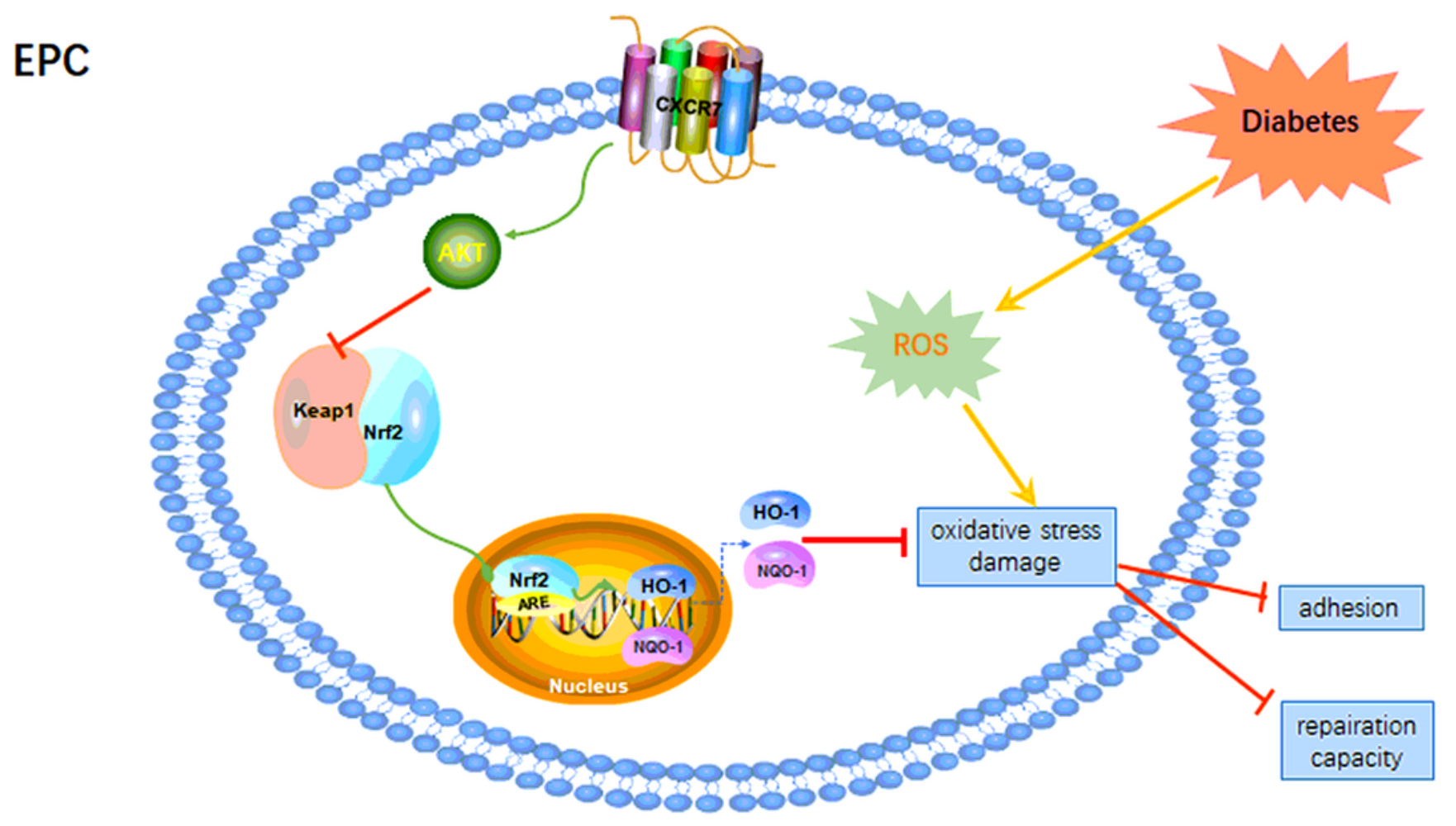

\section{Figure 7}

Illustration of the protective effect of the SDF-1/CXCR7 axis on diabetic EPCs. Diabetes decreased the expression of CXCR7 in diabetic EPCs. It induced impaired adhesion and reparation capacity of EPCs in vitro and in vivo. However, elevating CXCR7 expression enhanced the functional activity of EPCs via activating the Akt-associated Keap-1/Nrf2 axis counteracting to oxidative stress damage induced by reactive oxygen species in diabetes mellitus. 